\title{
Review
}

Ophthalmologica

Ophthalmologica 2019;242:1-7

Received: November 13, 2018

DOI: 10.1159/000499115

Accepted after revision: February 25, 2019

Published online: March 29, 2019

\section{Same-Day Bilateral Intravitreal Anti-Vascular Endothelial Growth Factor Injections: Experience of a Large Canadian Retina Center}

\author{
Verena R. Juncal ${ }^{a, b}$ Carolina L.M. Francisconi ${ }^{a, b}$ Filiberto Altomare ${ }^{a, b}$ \\ David R. Chow ${ }^{a-c}$ Louis R. Giavedoni ${ }^{a, b}$ Rajeev H. Muni ${ }^{a, b}$ Alan R. Berger ${ }^{a-c}$ \\ David T. Wong ${ }^{\mathrm{a}, \mathrm{b}, \mathrm{d}}$ \\ a Department of Ophthalmology, St. Michael's Hospital, Toronto, ON, Canada; ${ }^{b}$ Department of Ophthalmology and \\ Vision Sciences, University of Toronto, Toronto, ON, Canada; 'Toronto Retina Institute, North York, ON, Canada; \\ dDepartment of Ophthalmology, Oakville Trafalgar Hospital, Oakville, ON, Canada
}

\section{Keywords}

Antivascular endothelial growth factor - Ranibizumab .

Bevacizumab · Aflibercept - Intravitreal injection .

Endophthalmitis

\begin{abstract}
Objective: To evaluate the outcomes and complications of bilateral same-day intravitreal anti-vascular endothelial growth factor (anti-VEGF) injections. Methods: This is a single-center, retrospective study that included 524 eyes of 262 patients who received concomitant bilateral intravitreal anti-VEGF injections in 2016 at St. Michael's Hospital, Toronto. If any of the patients were receiving simultaneous bilateral injections on a regular basis prior to 2016, data pertaining to previous injections were also reviewed. Everyone received bevacizumab, ranibizumab, or aflibercept in an office setting. Results: A total of 9,798 intravitreal anti-VEGF injections (4,899 bilateral injection sessions) were performed in 524 eyes of 262 patients. The average number of bilateral injec-
\end{abstract}

tion sessions per patient was $18.7 \pm 14.1$. Ranibizumab was the most commonly used anti-VEGF drug (83.8\%). The incidence of endophthalmitis was $0.01 \%$, and there were 2 episodes of acute intraocular inflammation among the 9,798 injections $(0.02 \%)$. All 3 cases occurred after treatment with ranibizumab. There were 2 deaths $(0.76 \%)$ due to nonvascular causes but no vascular related systemic adverse events were reported. Conclusions: Same-day bilateral intravitreal anti-VEGF injections present a low rate of complications and are well tolerated by patients. This safe practice may reduce the burden on the health-care system and on the patients.

(c) 2019 S. Karger AG, Basel

Institution where study was performed: Department of Ophthalmology, St. Michael's Hospital, Toronto, ON, Canada.

Presentations: Oral presentation at Euretina 2017 (Barcelona, Spain). Poster presentations at American Society of Retinal Specialists (ASRS) Annual Meeting 2017 (Boston, MA, USA) and Association for Research in Vision and Ophthalmology (ARVO) Annual Meeting 2017 (Baltimore, MD, USA).

\section{KARGER}

(c) 2019 S. Karger AG, Basel

E-Mail karger@karger.com

www.karger.com/oph
David T. Wong, MD, FRCSC

Associate Professor of Ophthalmology and Vision Sciences, Department of Ophthalmology and Vision Sciences, Faculty of Medicine, University of Toronto St. Michael's Hospital, 801-61 Queen Street East, Toronto, ON, M5C 2T2 (Canada) E-Mail wongd@smh.ca 


\section{Introduction}

Intravitreal anti-vascular endothelial growth factor (anti-VEGF) agents are currently the first-line therapy for various retinal diseases, including neovascular age-related macular degeneration (nAMD) and diabetic macular edema (DME), since common growth factors and cytokines play a major role in their pathogenesis $[1,2]$. The number of intravitreal anti-VEGF injections performed worldwide has increased exponentially in the past decade, and multiple intravitreal injections over a period of months or years may be required to treat these diseases $[3,4]$. Even though less frequent dosing such as treat and extend or as-needed regimens have been proven to be efficient in various clinical trials, many patients frequently still require monthly injections $[1,5,6]$. In addition, patients often show up with bilateral eye involvement, and the rate of bilateral anti-VEGF treatment has been described as $36 \%$ for nAMD and $48 \%$ for DME [7-9]. Consequently, more frequent visits to the treating retinal specialist are required, increasing the burden on patient's time and productivity and the administrative load on the health-care system.

Administration of same-day bilateral injections instead of an alternating unilateral regimen is becoming a more common practice. In 2011, a survey done with retinal specialists in the United States revealed that 46\% performed bilateral simultaneous intravitreal injections [10]. However, some physicians do not feel comfortable treating both eyes on the same day fearing the local and systemic complications of anti-VEGF agents. The double number of visits required by those receiving an alternating unilateral treatment may reflect on a series of consequences for the patient and family members, including more hours off from work, lower productivity, and higher cost with transportation. For the offices, there are more visits and administrative work leading to less efficiency. Therefore, performing same-day bilateral injections likely results in better cost-effectiveness and greater patient's convenience, translating into benefits for patients and for the health-care system.

Studies evaluating the safety of same-day bilateral intravitreal injections have shown that they are well tolerated by patients and have a similar ocular complication profile as unilateral injections when separate instruments and medications are used for each eye. The overall rate of infectious endophthalmitis after intravitreal injection varies from 0.028 to $0.541 \%[11,12]$, and reports from bilateral same-day injections have shown similar results [13-18]. In terms of systemic safety, there is a concern that increased suppression of systemic VEGF levels resulting from simultaneous double dose of intravitreal anti-VEGF injections could lead to a higher risk of vascular adverse events, since plasma VEGF plays an important role regulating antiapoptotic pathways of the vessels' endothelial cells [19]. In this study, we report the ocular and systemic complications of same-day bilateral intravitreal anti-VEGF injections performed in a large tertiary retinal practice in Canada.

\section{Methods}

An electronic search of billing records was performed to identify patients who had received bilateral same-day intravitreal antiVEGF injections by a single retina group at St. Michael's Hospital, Toronto, between January 1, 2016, and December 31, 2016. A total of 262 patients were identified and their medical records were reviewed. If any of the identified patients were receiving simultaneous bilateral injections on a regular basis prior to 2016, data pertaining to these previous injections and follow-ups were also included in our analysis. This study was conducted according to the Declaration of Helsinki and approved by St. Michael's Hospital research ethics board.

The following data were collected from the patient's medical records: age, gender, ocular diagnosis, whether bevacizumab, ranibizumab, or aflibercept was used, number of bilateral same-day intravitreal injections received, Snellen best corrected visual acuity and intraocular pressure before each injection and need for anterior chamber paracentesis (AC tap) during injection visit. Medical records were also reviewed for postinjection adverse events: ocular complications, such as endophthalmitis, acute intraocular inflammation, and vitreous hemorrhage; occurrence of newly developed medical problems throughout follow-up, including myocardial infarction, cerebrovascular accident, and death; patient's request to switch to an alternating unilateral injection regimen due to intolerance of same-day bilateral injections. Patients were encouraged to call the office in case of any post-injection complaints. Even though a phone call log was not available for data collection, it was possible to identify in each chart whether there were any clinically relevant complaints noted by the patient that required an extra follow-up visit.

Bevacizumab, $1.25 \mathrm{mg} / 0.05 \mathrm{~mL}$; ranibizumab, $0.5 \mathrm{mg} / 0.05 \mathrm{~mL}$; and aflibercept, $2.0 \mathrm{mg} / 0.05 \mathrm{~mL}$ were injected by 6 vitreoretinal specialists (A.R.B., D.R.C., D.T.W., F.A., L.G., and R.H.M.) in an office setting. Aflibercept syringes were withdrawn from singleuse vials at the moment of injection. Bevacizumab injections consisted of prepackaged syringes from a compounding pharmacy. Ranibizumab injections included both prefilled syringes or were loaded from single-use vials. Each eye was injected separately in a sterile manner. All patients were prepared with topical tetracaine drops, followed by $5 \%$ povidone-iodine drops in both eyes. In addition, 3 physicians routinely performed subconjunctival anesthesia with $1 \%$ lidocaine in the quadrant to be injected. One single physician performed eyelid retraction using a bimanual eyelid retraction technique. All other 5 physicians routinely used sterile 
Table 1. Anti-VEGF injections used by the 262 study patients ( 524 eyes)

\begin{tabular}{lc}
\hline Characteristics & Total \\
\hline Initiating anti-VEGF agent, \% & \\
Ranibizumab & 83.8 \\
Aflibercept & 12.4 \\
Bevacizumab & 3.8 \\
Number of injections per anti-VEGF agent, $n(\%)$ & \\
Ranibizumab & $7,824(79.9)$ \\
Aflibercept & $1,860(19.0)$ \\
Bevacizumab & $114(1.1)$ \\
\hline
\end{tabular}

VEGF, vascular endothelial growth factor.

Table 2. Extra follow-up visits required by 13 patients

\begin{tabular}{|c|c|c|}
\hline Patients & $\begin{array}{l}\text { Extra } \\
\text { visits }\end{array}$ & Reason for visit \\
\hline 1 & 1 & Vitreous hemorrhage \\
\hline 2 & 1 & Floaters \\
\hline 3 & 1 & Subconjunctival hemorrhage \\
\hline 4 & 1 & $\begin{array}{l}\text { Visual loss due to worsening of diabetic macular } \\
\text { edema }\end{array}$ \\
\hline 5 & 1 & Floaters \\
\hline 6 & 9 & $\begin{array}{l}1 \text { episode in each eye of acute sterile intraocular } \\
\text { inflammation }\end{array}$ \\
\hline 7 & 1 & Floaters \\
\hline 8 & 3 & $\begin{array}{l}1 \text { visit: subconjunctival hemorrhage } \\
1 \text { visit: floaters } \\
1 \text { visit: mild conjunctival hyperemia }\end{array}$ \\
\hline 9 & 1 & Mild pain with normal exam \\
\hline 10 & 2 & $\begin{array}{l}1 \text { visit: floaters } \\
1 \text { visit: visual loss due to vitreous hemorrhage } \\
\text { secondary to ruptured arterial macroaneurysm }\end{array}$ \\
\hline 11 & 1 & Floaters \\
\hline 12 & 1 & Floaters \\
\hline 13 & 7 & Unilateral endophthalmitis \\
\hline
\end{tabular}

eyelid speculum for intravitreal injection procedures. The sterile eyelid speculum was placed initially in one eye, followed by an additional $5 \%$ povidone-iodine drop. The drug was injected 3.5 or 4.0 $\mathrm{mm}$ posterior to the limbus with a sterile syringe and needle. Two physicians used 32-gauge needles, while 4 used 30-gauge needles. The superotemporal quadrant was the elected site of injection for all surgeons, except for 1 who preferred the inferonasal quadrant. A cotton tip applicator was held at the site of the injection as the needle was withdrawn from the eye. The eyelid speculum was then removed from the first eye. The second eye was treated immediately after the first one using the same procedure described above, but with a new and separate tray of instruments and eye drops. Gloves and masks were not used, but conversation was avoided during the injection procedure. Patients did not receive pre-injec-

Same-Day Bilateral Anti-VEGF Injections tion or post-injection antibiotics on a regular basis. Lot numbers were not necessarily tracked, and it was not insisted that bilateral eyes received drugs belonging to different lot numbers.

Descriptive and statistical analyses were performed using IBM SPSS Statistics for Windows, version 24.0 (IBM Corporation, Armonk, NY, USA). Patients' characteristics are presented in terms of mean, SD, median, and range or percentage as appropriate. Mann-Whitney Test was used to compare continuous variables, and Pearson chi-square test was used to compare proportions. Statistical significance was set at $p<0.05$. The main outcome measure was the occurrence of ocular complications following treatment with same-day bilateral intravitreal injections.

\section{Results}

A total of 9,798 intravitreal anti-VEGF injections $(4,899$ bilateral injection sessions) were performed in 524 eyes of 262 patients. The mean age of the patients was $76.8 \pm 11.1$ years and most were female (57.6\%). The average number of bilateral injection sessions per patient was $18.7 \pm 14.1$ (range 1-71), and the mean follow-up time was $27.4 \pm 18.8$ months (range 1-76). The primary indications for anti-VEGF therapy were nAMD in $65.5 \%$ of the cases, DME in $32.9 \%$, cystoid macular edema in $0.4 \%$, polypoidal choroidal vasculopathy in $0.8 \%$, and macular edema secondary to retinal vein occlusion in $0.4 \%$. Ranibizumab was the most commonly used antiVEGF agent (Table 1). Ninety-six patients (36.6\%) had the initiating anti-VEGF agent switched throughout their follow-up.

Seventy-five patients (28.7\%) required an AC tap due to an episode or history of acute rise in intraocular pressure following intravitreal injections or due to uncontrolled glaucoma. A total of 501 AC taps (5.1\%) were performed among these 75 patients (mean: $6.7 \pm 5.9$ ). In total, 36 eyes of 18 patients (7.0\%) had a prior diagnosis of glaucoma, and 24 (66.7\%) of these eyes had at least $1 \mathrm{AC}$ tap done in the same visit of the intravitreal injection. The diagnosis of glaucoma was significantly associated with the need for an AC tap $(p=0.001)$.

Thirteen patients (4.9\%) required 30 extra follow-up visits $(0.6 \%)$ aside from their injection schedule due to clinically relevant complaints related to the intravitreal injections (Table 2). Following the 9,798 injections, there was one case $(0.01 \%)$ diagnosed as endophthalmitis in an 88 -year old female who had received 7 bilateral ranibizumab injections for nAMD with no previous complications. She presented with severe pain and acute vision loss in the right eye 6 days after the last injection. Her visual acuity worsened from counting fingers to hand motion, and clinical examination revealed conjunctival hyper- 
emia, a $0.5 \mathrm{~mm}$ hypopyon and vitritis. Vitreous and AC tap were performed followed by intravitreal injections of vancomycin $1.0 \mathrm{mg} / 0.1 \mathrm{~mL}$, ceftazidime $2.25 \mathrm{mg} / 0.1 \mathrm{~mL}$, and dexamethasone $0.4 \mathrm{mg} / 0.1 \mathrm{mg}$. Additional treatment included oral moxifloxacin, topical prednisolone $1.0 \mathrm{mg}$, vancomycin $50 \mathrm{mg} / \mathrm{mL}$, and tobramycin $14 \mathrm{mg} / \mathrm{mL}$. Both $\mathrm{AC}$ and vitreous specimens were culture negative, but there was an insufficient amount of vitreous sample for proper analysis according to the lab. The patient responded well to treatment: 2 weeks later, her vision was back to baseline counting fingers, and, 4 weeks after her symptoms onset, her clinical exam showed no more inflammatory signs. Same-day bilateral ranibizumab injections were resumed 2 months later with no further adverse events. In the meantime, there were no complications or inflammatory signs in the fellow eye.

There were 2 episodes of acute intraocular inflammation among the 9,798 injections. (0.02\%). An 80-year-old female receiving bilateral ranibizumab injections for nAMD presented 4 days later with a painless drop in vision from 20/100 to 20/400, no redness, AC cellular reaction $3+$, no hypopyon, and mild vitreous opacities in her left eye. After using topical prednisolone $1.0 \%$ for 4 days, her vision returned to preinjection level. Two months later, she experienced pain in the right eye 3 days after ranibizumab injection, with vision decreasing from $20 / 100$ to hand motion, a $1.0 \mathrm{~mm}$ hypopyon, and moderate vitritis. Despite her recent history of noninfectious acute intraocular inflammation following ranibizumab injection in the other eye, there was a raised suspicion for infectious endophthalmitis, leading the treating physician to collect $\mathrm{AC}$ and vitreous specimens for cultures. In addition, intravitreal vancomycin, ceftazidime, and dexamethasone were injected, and the patient was started on topical antibiotics and prednisolone $1.0 \%$. The patient experienced a rapid recovery within 3 days and cultures showed no growth. After being kept only on topical steroids for 2 weeks, her vision was completely recovered. Even though it was difficult to differentiate between infectious endophthalmitis from sterile intraocular inflammation, the clinical course suggested that both episodes were in fact a sterile, acute intraocular inflammation induced by the drug.

Other ocular adverse events directly related to the intravitreal injections included: one case of unilateral retinal tear detected 4 days after intravitreal ranibizumab and one case of unilateral mild vitreous hemorrhage 1 day after ranibizumab injection not explained by the underlying ocular disease and that spontaneously improved. None of the eyes developed retinal detachments.
None of the patients had vascular-related systemic adverse events following injections, such as cerebrovascular or thromboembolic events, during the follow-up period. There were 2 deaths $(0.76 \%)$ reported due to nonvascular causes.

\section{Discussion}

This large retrospective study reinforces that same-day bilateral intravitreal anti-VEGF injections are safe and well tolerated by patients. Our study differs from previous ones by including a greater number of bilateral injections than most series, by including a large population using all 3 anti-VEGF agents currently available (ranibizumab, bevacizumab, and aflibercept), and by analyzing not only rates of endophthalmitis but also of sterile intraocular inflammation and systemic adverse events based on detailed chart review. The significant increase in the number of intravitreal anti-VEGF injections in clinical practice and the rising need for bilateral treatment have resulted in more retinal specialists performing same-day bilateral injections. Undoubtedly, this practice is more cost-effective and convenient to the patients and to the health-system, but its safety profile should be an important concern.

Infectious endophthalmitis is the most feared ocular adverse event following intravitreal anti-VEGF injections and is a main concern regarding the safety of same-day bilateral injections. Many retinal specialists are uncomfortable to perform concomitant bilateral anti-VEGF injections fearing a higher risk of endophthalmitis. In this study, the incidence of endophthalmitis was $0.01 \%$ ( 1 case in 9,798 injections), which is near the lower end of the range described by previous reports $(0.0-0.065 \%)[13-18,20]$. Our study described a similar incidence while involving as much injections $(9,798)$ as some large clinical trials that have assessed the efficacy and safety of anti-VEGF therapy. In the MARINA study, for example, there were 5 cases of presumed endophthalmitis after 10,443 injections (0.05\%) [21] and, in the meta-analysis published by McCannel, the cumulative rate of endophthalmitis was $0.049 \%$ [11]. Recently, Borkar et al. [20] have also recently encountered a low rate $(0.027 \%)$ of endophthalmitis in the largest reported cohort of patients undergoing bilateral anti-VEGF injections.

Even though our retina group involves 6 vitreoretinal surgeons and each one has its own preferences regarding the techniques for intravitreal injection, some steps
Juncal/Francisconi/Altomare/Chow/ Giavedoni/Muni/Berger/Wong 
during the procedure are performed on a standard fashion among all of them. We believe that our low rate of endophthalmitis after bilateral injections is especially due to the fact that each injection is treated as a separate aseptic procedure, where new and separate speculum, cotton-tip applicator, syringe, and needles are used for each eye. Furthermore, application of 5\% povidone-iodine on the ocular surface before the injection is consistent among all physicians, since it is the only proven measure to prevent endophthalmitis $[22,23]$. One of the retinal specialists performs injections on the inferonasal quadrant and does not use an eyelid speculum, but, most importantly, contact between the eyelashes and the injection site is prevented with manual eyelid retraction. Indeed, neither eyelid speculum use nor site of injection has been shown to affect the risk of infection [24]. Our only case of presumed infectious endophthalmitis had an eyelid speculum placed, and injection was done in the superotemporal quadrant without prior subconjunctival lidocaine. All injections were done in an office setting, and none of the physicians used gloves, drapes, or masks. There is no definite data confirming that wearing gloves reduces the risk of infection, so, most importantly, handwashing or alcohol-based hand rubs before patient contact is done and careful attention is given not to touch the injection needle. Even though masks are not used, talking during the procedure is avoided, as supported by previous studies that showed that wearing masks and avoiding talking had a similar reduction in bacterial contamination $[25,26]$. In addition, our case of endophthalmitis did not receive subconjunctival anesthesia, but there are data suggesting that subconjunctival lidocaine $2 \%$ can have antibacterial properties and reduces the rate of infectious endophthalmitis [27]. Finally, pre and postinjection antibiotics are not routinely used in our practice, supported by data suggesting that the use of antibiotics does not lower the incidence of endophthalmitis and may contribute to development of a drug-resistant conjunctival flora [22, 23].

Sterile intraocular inflammation is an important issue associated with intravitreal anti-VEGF agents, and this study presents the largest number of bilateral anti-VEGF injections where this complication has been assessed. In our study, we had a $0.02 \%$ incidence of acute intraocular inflammation after ranibizumab injections, similar to previous series [14]. Interestingly, both episodes were reported in a single patient in different eyes and at distinct moments. To this date, the pathophysiology of sterile intraocular inflammation and the reason why patients receiving bilateral injections present with unilateral epi- sodes of acute intraocular inflammation remain unclear. Because the eye contains immune privileged sites, a possibility is that immune-related responses of the eye can react to antibody molecules after prior exposure to the drug [14]. Another possible explanation is related to the proinflammatory interaction of the crystallizable fragment $(\mathrm{Fc})$ component, present in aflibercept and bevacizumab, with intraretinal Fc receptors $[28,29]$. This theory could explain the higher rates of ocular inflammation following treatment with aflibercept when compared to ranibizumab [29]. In our study, there were no cases related to aflibercept, however this might be due to the smaller sample size of patients receiving aflibercept injections $(n=1,860)$, not being able to detect such rare event. Even though it is a rare situation that usually presents with complete visual recovery $[14,29]$, it can be difficult to differentiate from infectious endophthalmitis and may cause a negative effect on the patient. Some clinical characteristics might suggest the diagnosis of sterile inflammation over endophthalmitis, such as more rapid onset, anterior segment inflammation greater than posterior inflammation, and prompt improvement with topical steroids.

The systemic effect of anti-VEGF agents has been a concern since these drugs became first-line therapy for several intraocular conditions, even though only small amounts of the anti-VEGF drugs are released from the eye into the systemic circulation $[28,30]$. While intraocular VEGF is a critical mediator of angiogenesis involved in the pathogenesis of diseases such as nAMD, DME, and RVO (retinal vein occlusion), systemic VEGF acts as a vascular protective factor essential for maintaining the integrity and antithrombogenic properties of the endothelium [31]. Therefore, prolonged suppression of systemic VEGF levels can be associated with cerebrovascular and thromboembolic adverse events. Large clinical trials have reported rates of arterial thromboembolic events of $<1-7.8 \%,<1-6 \%,<1-6 \%,<1-5.8 \%$ for aflibercept, bevacizumab, ranibizumab, and control groups, respectively $[1,21,32,33]$. However, data regarding the systemic effect of bilateral anti-VEGF injections are still scarce. In our large series, there were no cardiovascular or thromboembolic events reported throughout patients' followup while being treated with bilateral anti-VEGF injections, suggesting that same-day bilateral treatment is systemically safe [16]. In addition, previous retrospective studies have shown that bilateral anti-VEGF injections did not increase the rate of systemic adverse events when compared to patients receiving unilateral injections, which is likely supported by the fact that the serum con- 
centration of VEGF is not significantly different between patients on bilateral or unilateral treatment $[15,16,34]$.

This study has some limitations, especially related to its retrospective design. Even though patients are routinely asked about new systemic and ocular events in their visits in this retina practice, we relied on the information provided by the patient and data in medical records filled out by physicians and their technicians. Furthermore, patients were encouraged to call the offices if there was any problem after injection but not all offices record this information in the patients' charts. Therefore, we observed which patients called the office and had clinically relevant symptoms that required extra follow-up visits based on detailed chart review. However, systemic adverse events could still have been missed based on this strategy. Since this study included patients receiving treatment by 6 vitreoretinal surgeons, the technique of intravitreal injection was not exactly the same for each patient. Even though a standard aseptic procedure in an office setting is performed by all, there are slight variations among their preferences that have been previously discussed. Finally, we can suggest but not draw definite conclusions about the safety of same-day bilateral compared to unilateral injections, since we did not have a control group of patients receiving unilateral injections for adequate comparison. Ideally, a prospective, randomized study evaluating the safety of concomitant bilateral anti-VEGF injections versus alternating unilateral injections should be performed.

In conclusion, same-day bilateral anti-VEGF intravitreal injections present a low rate of ocular and systemic complications. Our rates of endophthalmitis and sterile acute intraocular inflammation were comparable to studies where only unilateral injections were performed. We therefore emphasize that meticulous safety precautions should be equally taken for both eyes when employing this treatment modality. Finally, this practice is well tolerated by patients and might reduce the burden on the patient and health-care system.

\section{Acknowledgment}

None.

\section{Statement of Ethics}

This study was approved by St. Michael's Hospital research ethics board.

\section{Disclosure Statement}

The authors declare no conflicts of interest relevant to the present study.

\section{Funding Source}

This research did not receive any grant from funding agencies in the public, commercial, or not-for-profit sectors.

\section{Author Contributions}

V.R.J.: has contributed to the conception and design, acquisition of data, analysis and interpretation of data, and drafting the manuscript. C.L.M.F.: has contributed to the acquisition of data, analysis and interpretation of data, and drafting the manuscript. F.A., D.R.C., L.R.G., R.H.M., and A.R.B.: have contributed with conception and design, acquisition of data, and critically reviewing the manuscript. D.T.: Wong has contributed to the conception and design, acquisition of data, analysis and interpretation of data, and critically reviewing the manuscript.

\section{References}

1 Wells JA, Glassman AR, Ayala AR, Jampol LM, Aiello LP, Antoszyk AN, et al.; Diabetic Retinopathy Clinical Research Network. Aflibercept, bevacizumab, or ranibizumab for diabetic macular edema. N Engl J Med. 2015 Mar;372(13):1193-203.

2 Rosenfeld PJ, Brown DM, Heier JS, Boyer DS, Kaiser PK, Chung CY, et al.; MARINA Study Group. Ranibizumab for neovascular age-related macular degeneration. $N$ Engl J Med. 2006 Oct;355(14):1419-31.

3 Keenan TD, Wotton CJ, Goldacre MJ. Trends over time and geographical variation in rates of intravitreal injections in England. $\mathrm{Br} \mathrm{J}$ Ophthalmol. 2012 Mar;96(3):413-8.
4 Campbell RJ, Bronskill SE, Bell CM, Paterson JM, Whitehead M, Gill SS. Rapid expansion of intravitreal drug injection procedures, 2000 to 2008: a population-based analysis. Arch Ophthalmol. 2010 Mar;128(3):359-62.

5 Wykoff CC, Croft DE, Brown DM, Wang R, Payne JF, Clark L, et al.; TREX-AMD Study Group. Prospective trial of treat-and-extend versus monthly dosing for neovascular agerelated macular degeneration: TREX-AMD 1-year results. Ophthalmology. 2015 Dec; 122(12):2514-22.

6 Payne JF, Wykoff CC, Clark WL, Bruce BB, Boyer DS, Brown DM; TREX-DME Study Group. Randomized Trial of Treat and Ex- tend Ranibizumab with and without Navigated Laser for Diabetic Macular Edema: TREXDME 1 Year Outcomes. Ophthalmology. 2017 Jan;124(1):74-81.

7 Gonder JR, Walker VM, Barbeau M, Zaour N, Zachau BH, Hartje JR, et al. Costs and quality of life in diabetic macular edema: canadian burden of diabetic macular edema observational study (C-REALITY). J Ophthalmol. 2014;2014:939315.

8 Macular Photocoagulation Study Group. Fiveyear follow-up of fellow eyes of patients with age-related macular degeneration and unilateral extrafoveal choroidal neovascularization. Arch Ophthalmol. 1993 Sep;111(9):1189-99. 
9 Giocanti-Auregan A, Tadayoni R, Grenet T, Fajnkuchen F, Nghiem-Buffet S, DelahayeMazza C, et al. Estimation of the need for bilateral intravitreal anti-VEGF injections in clinical practice. BMC Ophthalmol. 2016 Aug;16(1): 142.

10 Green-Simms AE, Ekdawi NS, Bakri SJ. Survey of intravitreal injection techniques among retinal specialists in the United States. Am J Ophthalmol. 2011 Feb;151(2):329-32.

11 McCannel CA. Meta-analysis of endophthalmitis after intravitreal injection of anti-vascular endothelial growth factor agents: causative organisms and possible prevention strategies. Retina. 2011 Apr;31(4):654-61.

12 Fintak DR, Shah GK, Blinder KJ, Regillo CD, Pollack J, Heier JS, et al. Incidence of endophthalmitis related to intravitreal injection of bevacizumab and ranibizumab. Retina. 2008 Nov-Dec;28(10):1395-9.

13 Bakri SJ, Risco M, Edwards AO, Pulido JS. Bilateral simultaneous intravitreal injections in the office setting. Am J Ophthalmol. 2009 Jul; 148(1):66-9.e1.

14 Lima LH, Zweifel SA, Engelbert M, Sorenson JA, Slakter JS, Cooney MJ, et al. Evaluation of safety for bilateral same-day intravitreal injections of antivascular endothelial growth factor therapy. Retina. 2009 Oct;29(9):1213-7.

15 Mahajan VB, Elkins KA, Russell SR, Boldt HC, Gehrs KM, Weingeist TA, et al. Bilateral intravitreal injection of antivascular endothelial growth factor therapy. Retina. 2011 Jan; 31(1):31-5.

16 Davis RP, Schefler AC, Murray TG. Concomitant bilateral intravitreal anti-VEGF injections for the treatment of exudative age-related macular degeneration. Clin Ophthalmol. 2010 Jul;4(1):703-7.

17 Ruão M, Andreu-Fenoll M, Dolz-Marco R, Gallego-Pinazo R. Safety of bilateral sameday intravitreal injections of anti-vascular endothelial growth factor agents. Clin Ophthalmol. 2017 Feb;11:299-302.
18 Abu-Yaghi NE, Shokry AN, Abu-Sbeit RH Bilateral same-session intravitreal injections of anti-vascular endothelial growth factors. Int J Ophthalmol. 2014 Dec;7(6): 1017-21

19 Csaky K, Do DV. Safety implications of vascular endothelial growth factor blockade for subjects receiving intravitreal anti-vascular endothelial growth factor therapies. Am J Ophthalmol. 2009 Nov; 148(5):647-56.

20 Borkar DS, Obeid A, Su DC, et al. Endophthalmitis Rates after Bilateral Same-Day Intravitreal Anti-Vascular Endothelial Growth Factor Injections. Am J Ophthalmol. 2018 Oct;194:1-6.

21 Rosenfeld PJ, Brown DM, Heier JS, Boyer DS, Kaiser PK, Chung CY, et al.; MARINA Study Group. Ranibizumab for neovascular age-related macular degeneration. $\mathrm{N}$ Engl J Med. 2006 Oct;355(14):1419-31.

22 Fagan XJ, Al-Qureshi S. Intravitreal injections: a review of the evidence for best practice. Clin Exp Ophthalmol. 2013 Jul;41(5): 500-7.

23 Avery RL1, Bakri SJ, Blumenkranz MS, Brucker AJ, Cunningham ET Jr, D'Amico DJ, et. Intravitreal injection technique and monitoring: updated guidelines of an expert panel. Retina. 2014 Dec;34 Suppl 12:S1-S18.

24 Shah CP, Garg SJ, Vander JF, Brown GC, Kaiser RS, Haller JA; Post-Injection Endophthalmitis (PIE) Study Team. Outcomes and risk factors associated with endophthalmitis after intravitreal injection of anti-vascular endothelial growth factor agents. Ophthalmology. 2011 Oct;118(10):2028-34.

25 Wen JC, McCannel CA, Mochon AB, Garner OB. Bacterial dispersal associated with speech in the setting of intravitreous injections. Arch Ophthalmol. 2011 Dec;129(12):1551-4

26 Doshi RR, Leng T, Fung AE. Reducing oral flora contamination of intravitreal injections with face mask or silence. Retina. 2012 Mar; $32(3): 473-6$
27 Tustin A, Kim SJ, Chomsky A, Hubbard GB $3 \mathrm{rd}$, Sheng J. Antibacterial properties of $2 \%$ lidocaine and reduced rate of endophthalmitis after intravitreal injection. Retina. 2014 May;34(5):935-42.

28 Avery RL, Castellarin AA, Steinle NC, Dhoot DS, Pieramici DJ, See R, et al. Systemic pharmacokinetics following intravitreal injections of ranibizumab, bevacizumab or aflibercept in patients with neovascular AMD. Br J Ophthalmol. 2014 Dec;98(12):1636-41.

29 Souied EH, Dugel PU, Ferreira A, Hashmonay R, Lu J, Kelly SP. Severe ocular inflammation following ranibizumab or aflibercept injections for age-related macular degeneration: A retrospective claims database analysis. Ophthalmic Epidemiol. 2016;23(2):71-9.

30 Avery RL, Castellarin AA, Steinle NC, Dhoot DS, Pieramici DJ, See R, et al. Systemic Pharmacokinetics and Pharmacodynamics of Intravitreal Aflibercept, Bevacizumab, and Ranibizumab. Retina. 2017 Oct;37(10):1847-58.

31 Chen HX, Cleck JN. Adverse effects of anticancer agents that target the VEGF pathway. Nat Rev Clin Oncol. 2009 Aug;6(8):465-77.

32 Martin DF, Maguire MG, Fine SL, Ying GS, Jaffe GJ, Grunwald JE, et al.; Comparison of Age-related Macular Degeneration Treatments Trials (CATT) Research Group. Ranibizumab and bevacizumab for treatment of neovascular age-related macular degeneration: two-year results. Ophthalmology. 2012 Jul;119(7):1388-98.

33 Korobelnik JF, Do DV, Schmidt-Erfurth U, Boyer DS, Holz FG, Heier JS, et al. Intravitreal aflibercept for diabetic macular edema. Ophthalmology. 2014 Nov;121(11):2247-54.

34 Wang X, Sawada T, Sawada O, Saishin Y, Liu $\mathrm{P}$, Ohji M. Serum and plasma vascular endothelial growth factor concentrations before and after intravitreal injection of aflibercept or ranibizumab for age-related macular degeneration. Am J Ophthalmol. 2014 Oct; 158(4):738-744.e1. 